Journal of Network and Computer Applications, vol. 31, no. 1, pp. 32-46, January 2008.

\title{
Performance Comparisons of Load Balancing Algorithms for I/O- Intensive Workloads on Clusters
}

\author{
Xiao Qin \\ Department of Computer Science and Software Engineering \\ Auburn University, Auburn, AL 36849 \\ xqin@auburn.edu \\ http://www.eng.auburn.edu/ xqin
}

\begin{abstract}
Load balancing techniques play a critically important role in developing high-performance cluster computing platforms. Existing load balancing approaches are concerned with the effective usage of CPU and memory resources. Due to imbalance in disk I/O resources under I/O-intensive workloads, the previous CPU- or memory-aware load balancing schemes suffer significant performance drop. To remedy this deficiency, in this paper we propose a novel loadbalancing algorithm (hereinafter referred to as IOLB) for clusters, which aims at maintaining high resource utilization under a wide range of workload conditions. Specifically, IOLB is conducive to reducing the average slowdown of all parallel jobs submitted to a cluster by balancing load in disk resources. This can, in turn, not only achieve the effective usage of global disk resources but also reduce response times of I/O-intensive parallel jobs. To theoretically study the optimization of the IOLB algorithm, we qualitatively comparing IOLB with two conventional CPU- and memory-aware load-balancing schemes. We prove that when the workloads become CPU-intensive or memory-intensive in nature, IOLB gracefully degrades towards the existing load-balancing schemes. Experimental results based on trace-driven simulations demonstratively show that the IOLB algorithm significantly improves the resource utilization of a cluster under I/O-intensive workloads. Furthermore, our results confirm that IOLB is able to maintain the same level of performance as the two existing approaches, because IOLB improves CPU and memory utilization under CPU- and memory-intensive workloads.
\end{abstract}


Journal of Network and Computer Applications, vol. 31, no. 1, pp. 32-46, January 2008.

\section{Introduction}

Load balancing techniques play an important role in the design and development of highperformance clusters. A variety of load balancing schemes [1][3][12][17] can be used to improve performance of parallel and distributed systems clusters by assigning work, at run time, to computational nodes with under-utilized resources. Dynamic load-balancing schemes have been extensively investigated, primarily focusing on CPU [12][14], memory [1][28], network [3][8][24], or a combination of CPU and memory [32] resources. Although the existing loadbalancing schemes are effective in maintaining high utilization of resources, the previous approaches suffer significant performance drop under I/O-intensive workloads due to imbalance in disk $\mathrm{I} / \mathrm{O}$ resources. It is worth noting that disk $\mathrm{I} / \mathrm{O}$ resources become a performance bottleneck under I/O-intensive workloads, since the performance gap between CPU and disk I/O is widening. It is believed that a way of solving the disk I/O bottleneck problem is to leverage loadbalancing techniques to achieve effective usage of global disk resources in clusters.

In this paper we propose a novel load-balancing algorithm (hereinafter referred to as IOLB) for parallel jobs running on clusters. The IOLB algorithm aims at improving utilization of disk I/O, CPU, and memory resources in a cluster under a wide spectrum of workload. Specifically, IOLB is conducive to balancing load in a variety of resources, thereby reducing slowdowns of all parallel jobs submitted to a cluster. After qualitatively comparing IOLB with two conventional CPU- and memory-aware load-balancing schemes, we prove that IOLB gracefully degrades towards the existing load-balancing schemes if workloads become CPU- and memory-intensive, respectively. We conducted trace-driven simulations to show that the IOLB algorithm significantly improves the resource utilization of clusters under I/O-intensive workloads. 
Journal of Network and Computer Applications, vol. 31, no. 1, pp. 32-46, January 2008.

Moreover, our results confirm that IOLB improves CPU and memory utilization under CPU- and memory-intensive workloads and, therefore, IOLB can maintain the same level of performance as the two existing approaches.

The rest of the paper is organized as follows. Related work in the literature is briefly reviewed in the following section. Section 3 describes a generic model and the IOLB load-balancing algorithm for parallel jobs. Section 4 presents a qualitative comparison between IOLB and the two existing load-balancing schemes. To confirm the analytical comparison, in Section 5 we made use of trace-driven simulations to quantitatively evaluate performance of the IOLB algorithm and the alternative solutions. Finally, Section 6 summarizes the main contributions of this paper and comments on future research directions.

\section{Related Work}

In the past decade, load balancing techniques in the context of CPU and memory resources has been extensively studied in recent years. For example, Harchol-Balter and Downey studied a preemptive migration policy that is more effective than non-preemptive migration policies under CPU-intensive workloads [12]. Zhang et al. [32] proposed new load sharing policies that are concerned with effective usage of both CPU and memory resources. The above load-balancing schemes are able to achieve high system performance under CPU- and memory-intensive workload conditions, respectively.

A number of approaches to balancing load in disk $\mathrm{I} / \mathrm{O}$ resources can be found in the literature [16][33]. Lee et al. studied two file assignments algorithms to balance load across all disks, thereby making it possible to improve overall system performance by fully utilizing available hard drives [16]. Zhang et al. proposed three I/O-aware scheduling schemes that are aware of the 
Journal of Network and Computer Applications, vol. 31, no. 1, pp. 32-46, January 2008.

job's spatial preferences [33].

In recent years, the issue of leveraging $\mathrm{I} / \mathrm{O}$ cache and buffer to boost performance of storage systems has been reported in the literature. Choi et al. proposed a buffer replacement scheme for effective caching of disk blocks[7]. Forney et al. investigated storage-aware caching algorithms in heterogeneous clusters [11]. Ma et al. developed an active buffering mechanism to alleviate the disk I/O bottleneck problem using local idle memory and overlapping I/O with computation [18]. We proposed a feedback control mechanism to improve the performance of a cluster by adaptively manipulating the I/O buffer size [23]. Our IOLB load-balancing algorithm is complementary to the aforementioned caching and buffering techniques, meaning that IOLB can provides additional performance improvement when the existing caching and buffering mechanisms.

\section{An I/O-aware Load-Balancing Algorithm}

\subsection{A generic model}

A cluster computing platform considered in this study consists of a set $N=\left\{N_{1}, N_{2}, \ldots, N_{n}\right\}$ of $n$ homogeneous nodes connected by a high-speed interconnection network like Myrinet. Note that the terms node and machine are used interchangeably throughout this paper. Throughout this paper, $N_{i}$ represents a set of tasks running on the $i$ th node. Each node in a cluster is composed of a combination of various resources, including processors, memory, network connectivity, and disks. A load manager residing in each node is responsible for load balancing and monitoring available resources of the node. Each job is associated to a home machine, through which the job is submitted to the cluster. A node becomes the home machine of a job either because the job is initially created on the node or because data to be accessed by the job is stored in the node. A 
Journal of Network and Computer Applications, vol. 31, no. 1, pp. 32-46, January 2008.

similar home model was proposed by Lavi and Barak in context of load balancing [15]. In the case that a job is submitted through its home node, the corresponding load manager for the home node is invoked to allocate the job to a node or a group of nodes with the least load. At any time, a node is either having its load manager performed or executing a task. When the load manager is carried out, the underlying computation may be concurrently performed or suspended. It is feasible to make the load manager and other tasks executed in parallel, since the load manager can be running in the background by an inexpensive coprocessor [31]. In addition, it is reasonable to assume that all load managers in a cluster is capable of keeping track of global load information by monitoring local resources and sharing load information through a direct communication network [22].

In this study we are concerned with a class of embarrassing parallel applications, each of which is represented in form of a set $T=\left\{\tau_{1}, \tau_{2}, \ldots, \tau_{m}\right\}$ of tasks (also referred to as processes) that are independent of one another. Some real-world examples of embarrassing parallel applications can be found in [29]. It is worth noting that the proposed load-balancing algorithm can be readily integrated with a communication load balancer to deal with parallel applications with dependent tasks. A task $\tau_{i}$ in $T$ is modeled as a tuple $\left(c_{i}, s_{i}, \lambda_{i}, d_{i}\right)$, where $c_{i}$ is the computational time, $s_{i}$ is the requested memory space measured by MBytes, $\lambda_{i}$ is the arrival rate of disk requests measured by number of disk accesses per ms (No./ms), and $d_{i}$ is the disk request's data size in KBytes. Note that the parameters $c_{i}$ and $s_{i}$ are used to describe task $\tau_{i}$ 's requirements for $\mathrm{CPU}$ and memory, whereas the $\mathrm{I} / \mathrm{O}$ requirement of $\tau_{i}$ is characterized by the other two parameters $-\lambda_{i}$ and $d_{i}$.

\subsection{Problem Formulation}


Journal of Network and Computer Applications, vol. 31, no. 1, pp. 32-46, January 2008.

Given a task $\tau_{i}$, we denote $t_{i}^{\text {ded }}$ as the time to execute the task on a dedicated computing cluster, and $t_{i}$ as the time to execute the task on the same cluster in a time-sharing setting. In our model, we consider three resources: CPU, memory, and disk I/O. Let $t_{i}^{\text {ded,CPU }}, t_{i}^{\text {ded,page }}$, and $t_{i}^{\text {ded,IO }}$ be the times of a task spent on CPU, page fault handling, and disk I/O processing in a dedicated mode. The values of $t_{i}^{\text {ded,CPU }}, t_{i}^{\text {ded,page }}$, and $t_{i}^{\text {ded,IO }}$ can be respectively derived from $\tau_{i}$ 's requirement parameters, including $c_{i}, s_{i}, \lambda_{i}$, and $d_{i}$. Let $t_{i}^{C P U}, t_{i}^{\text {page }}$, and $t_{i}^{I O}$ denote the times spent on the three resources in a time-shared mode. Since tasks running on computational nodes may be delayed by contention for resources, the slowdown imposed on task $\tau_{i}$ is expressed as the ratio between the task's execution time in the time-shared mode and its execution time on the same cluster in the dedicated mode. Thus, the slowdown of $\tau_{i}$ is written as

$$
s d_{i}=\frac{t_{i}}{t_{i}^{\text {ded }}}=\frac{t_{i}^{C P U}+t_{i}^{\text {page }}+t_{i}^{I O}}{t_{i}^{\text {ded,CPU }}+t_{i}^{\text {ded,page }}+t_{i}^{\text {ded,IO }}} .
$$

Given a parallel application with task set $T_{i}=\left\{\tau_{1}, \tau_{2}, \ldots, \tau_{m i}\right\}$, the slowdown of the application is calculated as the average slowdown of all the independent tasks in $T$. Thus, the slowdown of the parallel application is written as

$$
s d\left(T_{i}\right)=\frac{1}{m_{i}} \sum_{j=1}^{m_{i}} s d_{j}=\frac{1}{m_{i}} \sum_{j=1}^{m_{i}} \frac{t_{j}^{C P U}+t_{j}^{\text {page }}+t_{j}^{I O}}{t_{j}^{\text {ded,CPU }}+t_{j}^{\text {ded,page }}+t_{j}^{\text {ded,IO }} .}
$$

For a special case where all the independent tasks in the set $T_{i}$ are identical, (i.e., $\left.\forall 1 \leq j \leq m_{i}: t_{j}^{\text {ded,CPU }}=t^{\text {ded,CPU }}, t_{j}^{\text {ded,page }}=t^{\text {ded,page }}, t_{j}^{\text {ded,IO }}=t^{\text {ded,IO }}\right)$, Eq. (2) can be rewritten as follows

$$
s d\left(T_{i}\right)=\frac{1}{m_{i} \cdot\left(t^{\text {ded,CPU }}+t^{\text {ded,page }}+t^{\text {ded,IO}}\right)} \sum_{j=1}^{m_{i}} t_{j}^{C P U}+t_{j}^{\text {page }}+t_{j}^{I O} .
$$


Journal of Network and Computer Applications, vol. 31, no. 1, pp. 32-46, January 2008.

The goal of the proposed load-balancing algorithm is to reduce the average slowdown of all parallel applications submitted to a cluster. This can, in turn, minimize the average response time of the running applications. Specifically, our algorithm aims at optimizing the following average slowdown of a sequence of parallel applications (e.g., $T_{1}, T_{2}, \ldots, T_{q}$ ) executed on a cluster

$$
\operatorname{minimize} s d=\frac{1}{q} \sum_{i=1}^{q} s d\left(T_{i}\right)=\frac{1}{q} \sum_{i=1}^{q}\left(\frac{1}{m_{i}} \sum_{j=1}^{m_{i}} \frac{t_{j}^{C P U}+t_{j}^{\text {page }}+t_{j}^{I O}}{t_{j}^{\text {ded,CPU }}+t_{j}^{\text {ded,page }}+t_{j}^{\text {ded }, I O}}\right) .
$$

\subsection{A load-balancing algorithm for I/O-intensive workloads}

Now we present a load-balancing algorithm (hereinafter referred to as IOLB) for a wide variety of workload conditions including I/O-intensive, CPU-intensive, and memory-intensive, workloads. The objective of the proposed IOLB algorithm is to balance the load of three types of resources across all nodes in a cluster such that the average slowdown of submitted parallel jobs is minimized. Since the goal of this study is to analytically evaluate the performance of the IOLB algorithm, we are focused on a remote execution mechanism in which a task can be running on a remote node where it started execution. Thus, preemptive migrations of tasks are not supported in the IOLB algorithm. Nevertheless, the IOLB load-balancing algorithm can be readily integrated with a preemptive migration mechanism, thereby providing further performance improvement. Recently, we studied a load-balancing algorithm with preemptive migration, and details of this algorithm can be found in [22].

To facilitate the description of the IOLB algorithm, we first introduce the following three load indices with respect to CPU, memory, and I/O resources. The CPU load index $L_{i}^{C P U}$ of node $i$ is defined as the sum of remaining CPU lifetimes of tasks running on the node. Thus, $L_{i}^{C P U}$ is

expressed as $L_{i}^{C P U}=\sum_{\tau_{j} \in N_{i}} r_{j}$, where $r_{j}$ is the expected remaining CPU lifetime of task $\tau_{i}$. The 
Journal of Network and Computer Applications, vol. 31, no. 1, pp. 32-46, January 2008.

memory load index $L_{i}^{\text {page }}$ of node $i$ is defined as the sum of page fault processing times $t_{j}^{\text {page }}$ of tasks on node $i$. Hence, we have $L_{i}^{\text {page }}=\sum_{\tau_{j} \in N_{i}} t_{j}^{\text {page }}$. Similarly, the I/O load index $L_{i}^{I O}$ of node $i$ is the sum of $\mathrm{I} / \mathrm{O}$ processing times of tasks on the node. Therefore, the $\mathrm{I} / \mathrm{O}$ load index can be written as

$$
L_{i}^{I O}=\sum_{\tau_{j} \in N_{i}}\left(r_{j} \cdot \lambda_{j} \cdot t_{j, I O}\right),
$$

where $t_{j, I O}$ is task $\tau_{j}$ 's the I/O processing time of each disk request. The value of $t_{j, I O}$ in Eq. (5) is computed by

$$
t_{i, I O}=t_{\text {seek }}+t_{r o t}+\frac{d_{i}}{B_{d i s k}},
$$

where $t_{\text {seek }}$ and $t_{r o t}$ are the seek time and rotational latency, and $\frac{d_{i}}{B_{\text {disk }}}$ is the data transfer time depending on data size $d_{i}$ and disk bandwidth $B_{\text {disk }}$.

In light of the three load indices described above, we propose a new concept of load imbalance factor to quantify the amount of imbalance in a cluster. The load imbalance factor of a resource is a product of the fraction of time spent on using the resource in a cluster and the discrepancy between the maximum and the minimum loads of the resource among all nodes in the cluster. More specifically, the load imbalance factors for CPU, memory, and I/O resources can be written as Eq. (7)-(9).

$$
L I F_{C P U}=\frac{\sum_{i=1}^{n} L_{i}^{C P U}}{\sum_{i=1}^{n} L_{i}^{C P U}+\sum_{i=1}^{n} L_{i}^{\text {page }}+\sum_{i=1}^{n} L_{i}^{I O}} \cdot \sum_{i=1}^{n}\left|L_{i}^{C P U}-\bar{L}_{i}^{C P U}\right|,
$$


Journal of Network and Computer Applications, vol. 31, no. 1, pp. 32-46, January 2008.

$$
\begin{aligned}
& L I F_{\text {page }}=\frac{\sum_{i=1}^{n} L_{i}^{\text {page }}}{\sum_{i=1}^{n} L_{i}^{C P U}+\sum_{i=1}^{n} L_{i}^{\text {page }}+\sum_{i=1}^{n} L_{i}^{I O}} \cdot \sum_{i=1}^{n}\left|L_{i}^{\text {page }}-\bar{L}_{i}^{\text {page }}\right|, \\
& L I F_{I O}=\frac{\sum_{i=1}^{n} L_{i}^{I O}}{\sum_{i=1}^{n} L_{i}^{C P U}+\sum_{i=1}^{n} L_{i}^{\text {page }}+\sum_{i=1}^{n} L_{i}^{I O}} \cdot \sum_{i=1}^{n}\left|L_{i}^{I O}-\bar{L}_{i}^{I O}\right|,
\end{aligned}
$$

where $\bar{L}_{i}^{C P U}=\frac{1}{n} \sum_{i=1}^{n} L_{i}^{C P U}, \bar{L}_{i}^{\text {page }}=\frac{1}{n} \sum_{i=1}^{n} L_{i}^{\text {page }}$, and $\bar{L}_{i}^{I O}=\frac{1}{n} \sum_{i=1}^{n} L_{i}^{I O}$. On the right-hand sides of Eqs. (7)-(9), the first terms, which reflect the importance of the three types of resources, are the fractions of times spent on computing, page fault handling, and disk I/O processing, respectively. The second terms on the right-hand side of Eqs. (7)-(9) are used to measure the amount of imbalance in the three resources.

The load imbalance factor LIF of a cluster can be derived from Eqs. (7)-(9) as the sum of the load imbalance factors of the three resource types. Thus, we have

$$
L I F=L I F_{C P U}+L I F_{\text {page }}+L I F_{I O} .
$$

Now we are positioned to delineate the IOLB load-balancing algorithm, of which the pseudocode is shown in Fig. 1. Given an embarrassing parallel application with a set $T$ of independent tasks submitted to a local node $N_{i}$ of a cluster, the IOLB algorithm make an effort to balance workload of the cluster's resources by allocating each task in $T$ to a computational node such that the task's expected response time (also known as turn around time) is minimized. In other words, IOLB aims to redistribute load among all the node in a cluster, thereby allowing the submitted parallel application to efficiently run on the cluster.

For each task $\tau_{j}$ in set $T$, the IOLB algorithm repeatedly performs Steps 2-12 described as 
Journal of Network and Computer Applications, vol. 31, no. 1, pp. 32-46, January 2008.

follows. First, the response time $R_{j}^{i}$ of $\tau_{j}$ on the local node $N_{i}$ is approximated by Step 2. The estimation of $R_{j}^{i}$ is important because it will be used to justify whether a remote execution of $\tau_{j}$ is worthwhile (see Steps 6, 11, and 16).

Algorithm: IO-Aware Load Balancing (IOLB)

Input: A local node $N_{i}$, a job with task set $T$ submitted to $N_{i}$.

1. for (each task $\tau_{j} \in T$ ) do

2. calculate the response time $R_{j}^{i}$ of $\tau_{j}$ on node $N_{i}$;

3. if $c_{j} \cdot \lambda_{j} \cdot t_{j, I O}>0$ and $L I F_{I O}=\max \left(L I F_{C P U}, L I F_{p a g e}, L I F_{I O}\right)$ and $L_{i}^{I O}=\max _{a=1}^{n}\left(L_{a}^{I O}\right)$ then

4. $\quad$ choose node $N_{k}$ such that $L_{k}^{I O}=\min _{a=1}^{n}\left(L_{a}^{I O}\right)$;

5. calculate the response time $R_{j}^{k}$ of $\tau_{j}$ on node $N_{k}$;

6. if $c_{j} \cdot \lambda_{j} \cdot t_{j, I O}<\left(c_{j} \cdot \lambda_{j} \cdot t_{j, I O}+L_{i}^{I O}-L_{k}^{I O}\right) / 2$ and $R_{j}^{i}>R_{j}^{k}+e$ then

7. dispatch task $\tau_{j}$ to node $N_{k}$ and remotely execute $\tau_{j}$ on $N_{k}$;

8. $\quad$ else locally execute $\tau_{j}$ on $N_{i}$;

9. else if $t_{j}^{\text {page }}>0$ and $L I F_{\text {page }}>L I F_{C P U}$ and $L_{i}^{\text {page }}=\max _{a=1}^{n}\left(L_{a}^{\text {page }}\right)$ then

10. $\quad$ choose node $N_{k}$ such that $L_{k}^{\text {page }}=\min _{a=1}^{n}\left(L_{a}^{\text {page }}\right)$;

11. calculate the response time $R_{j}^{k}$ of $\tau_{j}$ on node $N_{k}$;

12. $\quad$ if $t_{j}^{\text {page }}<\left(t_{j}^{\text {page }}+L_{i}^{\text {page }}-L_{k}^{\text {page }}\right) / 2$ and $R_{j}^{i}>R_{j}^{k}+e$ then

13. dispatch task $\tau_{j}$ to node $N_{k}$ and remotely execute $\tau_{j}$ on $N_{k}$;

14. $\quad$ else locally execute $\tau_{j}$ on $N_{i}$;

15. else if $L_{i}^{C P U}=\max _{a=1}^{n}\left(L_{a}^{C P U}\right)$ then

16. $\quad$ choose node $N_{k}$ such that $L_{k}^{C P U}=\min _{a=1}^{n}\left(L_{a}^{C P U}\right)$;

17. $\quad$ calculate the response time $R_{j}^{k}$ of $\tau_{j}$ on node $N_{k}$;

18. if $r_{j}<\left(r_{j}+L_{i}^{C P U}-L_{k}^{C P U}\right) / 2$ and $R_{j}^{i}>R_{j}^{k}+e$ then

19. $\quad$ dispatch task $\tau_{j}$ to node $N_{k}$ and remotely execute $\tau_{j}$ on $N_{k}$;

20. $\quad$ else locally execute $\tau_{j}$ on $N_{i}$;

21. Update the load status;

22.end for

Fig. 1. Pseudocode of the IOLB load-balancing algorithm. 
Journal of Network and Computer Applications, vol. 31, no. 1, pp. 32-46, January 2008.

Second, Step 3 is responsible for initiating the process of balancing workload of disk I/O resources. Specifically, Steps 4-7 are invoked to balance the load of I/O resources in the case that all the following three conditions hold in Step 3. Condition 1 (i.e., $c_{j} \cdot \lambda_{j} \cdot t_{j, I O}>0$ ) states that the I/O load of $\tau_{j}$ must be greater than zero (see Theorems 2 and 3). Condition 2 (i.e., $\left.L I F_{I O}=\max \left(L I F_{C P U}, L I F_{\text {page }}, L I F_{I O}\right)\right)$ says that the load imbalance factor of disk I/O must be higher than those CPU and memory resources. Condition 3 (i.e., $L_{i}^{I O}=\max _{a=1}^{n}\left(L_{a}^{I O}\right)$ ) means that the I/O load of the local node is the highest among those of all the nodes.

Third, if it becomes a necessity to balance I/O load, then Step 4 chooses the most approximate remote node $N_{k}$ with the lightest load with respect to disk $\mathrm{I} / \mathrm{O}$, followed by estimating the response time $R_{j}^{k}$ of $\tau_{j}$ on the candidate node $N_{k}$. Step 6 is of critical importance to ensure performance improvement achieved by having $\tau_{j}$ executed remotely. More specifically, before Step 7 dispatches $\tau_{j}$ and has it remotely executed on $N_{k}$, Step 6 must make sure that the following two conditions are satisfied. Condition 1 (i.e., $c_{j} \cdot \lambda_{j} \cdot t_{j, I O}<$ $\left(c_{j} \cdot \lambda_{j} \cdot t_{j, I O}+L_{i}^{I O}-L_{k}^{I O}\right) / 2$ ) guarantees that the load discrepancy between $L_{i}^{I O}$ and $L_{k}^{I O}$ is reduced. Condition 2 (i.e., $R_{j}^{i}>R_{j}^{k}+e$, where $e$ is the remote execution overhead) ensures that the expected response time of $\tau_{j}$ on the selected remote node $N_{k}$ is less than the response time of $\tau_{j}$ on the local node $N_{i}$. In the case that the remote execution of $\tau_{j}$ is not beneficial, Step 8 has $\tau_{j}$ locally executed on $N_{i}$.

Fourth, Step 9 decides if the following three conditions are satisfied before a meaningful 
Journal of Network and Computer Applications, vol. 31, no. 1, pp. 32-46, January 2008.

remote execution is performed. Condition 1 (i.e., $t_{j}^{\text {page }}>0$ ) says that $\tau_{j}$ must exhibit page fault behavior (see Theorems 5 and 6). Page fault behaviors occur when the memory space required by running tasks exceeds the amount of available memory space. Condition 2 (i.e., $\left.L I F_{\text {page }}>L I F_{C P U}\right)$ indicates that the load imbalance factor of memory resources must be higher than that CPU resources. Condition 3 (i.e., $L_{i}^{\text {page }}=\max _{a=1}^{n}\left(L_{a}^{\text {page }}\right)$ ) states that the load of page-fault processing in the local node is the highest among those of all the nodes. If the above conditions hold, Steps 10-14 aim to balance the load of memory resources by transferring task $\tau_{j}$ from the overloaded node to a remote node that are lightly loaded with respect to memory. Step 12 is carried out to guarantee that the remote execution of $\tau_{j}$ leads to performance improvement.

Fifth, if there is no way of balancing the disk I/O and memory resources in the cluster, Steps 15-20 attempt to evenly distribute the CPU load. When the local node is overloaded with respect to CPU resource (See Step 15), task $\tau_{j}$ is dispatched to and executed by a remote node with the lightest CPU load. Step 19 makes the remote execution possible if such a remote execution is beneficial (see Step 18).

Last, Step 21 maintains updated load information that is broadcasted to the local node and other nodes in the cluster.

The following theorem proves the time complexity of the IOLB load-balancing algorithm. Theorem 1. Given a cluster and a parallel application submitted to the cluster, the time complexity of the IOLB algorithm is $O(n m)$, where $n$ is the number of nodes in the cluster, $m$ is the number of tasks in the application, and the values of $n$ and $m$ are much larger than 2 .

Proof. It takes $O(1)$ time to compute the response time of a task on a node. The time complexity 
Journal of Network and Computer Applications, vol. 31, no. 1, pp. 32-46, January 2008.

of determining that a local node is overly loaded is $O(n)$, since there are $n$ nodes in the cluster (see Step 3). Step 4 takes $O(n)$ time to choose the most appropriate node with the minimal load. Steps 6 and 7 take $O(1)$ time. Hence, the time complexity of balancing disk I/O resources is $O(2+2 n)$ (see Steps 7-12). Similarly, the time complexities of balancing memory and CPU resources are both $O(2+2 n)$. Since there are $\mathrm{m}$ tasks in the parallel application, the time complexity of the IOLB algorithm is $O(2+2 n) O(m)=O(2(1+n) m)$. The values of $n$ and $m$ in most cases are much larger than 2 and, therefore, the time complexity becomes $O(n m)$.

\section{An Analytical Comparison}

In this section, we first prove important properties of the IOLB algorithms (see Lemmas 1-2, Theorems 2-6). Next, we qualitatively compare IOLB with two existing load-balancing algorithms (see Theorems 7 and 9).

\subsection{Properties}

Theorem 2. Let $c_{j}, \lambda_{j}$, and $t_{j, I O}$ be the execution time, I/O arrival rate of task $\tau_{j}$, and the I/O processing time of each disk request. If the value of $c_{j} \cdot \lambda_{j} \cdot t_{j, I O}$ is zero, then the allocation of $\tau_{j}$ has no impact on balancing disk I/O resources.

Proof. Before the allocation of $\tau_{j}$, the amount of imbalance with respect to disk I/O resources can be measured by $\sum_{i=1}^{n}\left|L_{i}^{I O}-\bar{L}_{i}^{I O}\right|$, where $\bar{L}_{i}^{I O}=\frac{1}{n} \sum_{i=1}^{n} L_{i}^{I O}$. Without loss of generality, we assume $\tau_{j}$ is allocated to $N_{k}$, and prior to the arrival of $\tau_{j}$ the I/O load of $N_{k}$ is $L_{i}^{I O}=\sum_{\tau_{j} \in N_{i}}\left(r_{j} \cdot \lambda_{j} \cdot t_{j, I O}\right)$ (see Eq. 5). The disk I/O load index after dispatching task $\tau_{j}$ to node $N_{k}$ becomes 
Journal of Network and Computer Applications, vol. 31, no. 1, pp. 32-46, January 2008.

$L_{i}^{\prime O}=c_{j} \cdot \lambda_{j} \cdot t_{j, I O}+\sum_{\tau_{j} \in N_{i}}\left(r_{j} \cdot \lambda_{j} \cdot t_{j, I O}\right)$. Since the value of $c_{j} \cdot \lambda_{j} \cdot t_{j, I O}$ is zero, we have $L_{i}^{\prime I O}=c_{j} \cdot \lambda_{j} \cdot t_{j, I O}+\sum_{\tau_{j} \in N_{i}}\left(r_{j} \cdot \lambda_{j} \cdot t_{j, I O}\right)=\sum_{\tau_{j} \in N_{i}}\left(r_{j} \cdot \lambda_{j} \cdot t_{j, I O}\right)=L_{i}^{I O}$. Thus, the values of $L_{i}^{I O}$ and $L_{i}^{\prime I O}$ are identical, meaning that the allocation of $\tau_{j}$ has no impact on balancing disk I/O resources.

Corollary 1. If the I/O arrival rate $\lambda_{j}$ of task $\tau_{j}$ is zero, then the allocation of $\tau_{j}$ has no impact on balancing disk I/O resources.

Proof. The value of $c_{j} \cdot \lambda_{j} \cdot t_{j, I O}$ becomes zero if the $\mathrm{I} / \mathrm{O}$ arrival rate $\lambda_{j}$ of task $\tau_{j}$ is zero. Then, the proof is immediate from Theorem 1.

Theorem 3. Suppose there is a task $\tau_{j}$ (initially submitted to node $N_{i}$ ) to be allocated in a cluster; the disk I/O processing time of $\tau_{j}$ is $c_{j} \cdot \lambda_{j} \cdot t_{j, I O}$. Then $c_{j} \cdot \lambda_{j} \cdot t_{j, I O}>0$ is a necessary condition for allocating task $\tau_{j}$ in a way to balance load with respect to disk I/O.

Proof. To prove the correctness of Theorem 3, we have to show that allocating $\tau_{j}$ in a way to balance load in disk $\mathrm{I} / \mathrm{O}$ that $c_{j} \cdot \lambda_{j} \cdot t_{j, I O}$ is larger than 0 . This can be proved by contradiction and, hence, let us assume that $t_{j}^{\text {page }}$ equals to 0 . Since $c_{j} \cdot \lambda_{j} \cdot t_{j, I O}=0$, Theorem 2 shows that the allocation of $\tau_{j}$ has no impact on balancing disk I/O resources, meaning that there is no way of allocating $\tau_{j}$ such that the imbalance load in disk I/O is alleviated. Hence, we obtain the contradiction that completes the proof.

Theorem 4. Let $t_{j}^{\text {page }}$ and $L_{j, m e m}$ be the page fault processing time and memory requirement of $\operatorname{task} \tau_{j}$. Let $L_{i}^{m e m}$ denote the accumulation of the memory space allocated to tasks running on the 
Journal of Network and Computer Applications, vol. 31, no. 1, pp. 32-46, January 2008.

node $N_{i}$. Thus, we have $L_{i}^{m e m}=\sum_{j \in N_{i}} L_{j, m e m}$. The page fault processing time $t_{j}^{\text {page }}$ is computed as,

$$
t_{j}^{\text {page }}=\left\{\begin{array}{c}
0, \quad \text { if } L_{i}^{\text {mem }} \leq M_{i}, \\
p_{i} \cdot \frac{\sum_{j \in N_{i}} L_{j, m e m}}{M_{i}} \cdot r_{j} \cdot\left(t_{\text {seek }}+t_{\text {rot }}+\frac{d_{\text {page }}}{B_{\text {disk }}}\right), \text { otherwise },
\end{array}\right.
$$

where $p_{i}$ is the page fault rate, $M_{i}$ is the total memory space available on node $N_{i}, r_{j}$ is the expected remaining CPU lifetime, $t_{\text {seek }}$ and $t_{\text {rot }}$ are the seek time and rotational latency, $d_{\text {page }}$ is the page size, and $\frac{d_{\text {page }}}{B_{\text {disk }}}$ is the data transfer time.

Proof. First, we have to prove that $t_{j}^{\text {page }}$ is zero if $L_{i}^{m e m} \leq M_{i}$. When the total available memory space $M_{i}$ can meet the memory demands of tasks running on the $i$ th node (i.e., $L_{i}^{m e m}<M_{i}$ ), no page fault occurs in the node. In this case $\tau_{j}$ exhibits no page fault behavior, and the page fault processing time $t_{j}^{\text {page }}$ is zero.

Second, let us prove that $t_{j}^{\text {page }}=p_{i} \cdot \frac{\sum_{j \in N_{i}} L_{j, m e m}}{M_{i}} \cdot r_{j} \cdot\left(t_{\text {seek }}+t_{\text {rot }}+\frac{d_{\text {page }}}{B_{\text {disk }}}\right)$ if $L_{i}^{\text {mem }}>M_{i}$. If $L_{i}^{\text {mem }}$ is larger than $M_{i}$, then the node encounters page faults. The number of page faults $\pi_{j}$ is proportional to (1) the page fault rate $p_{i},(2)$ the accumulated memory space $L_{i}^{m e m}$ allocated to all the running tasks on $N_{i}$, and (3) the expected remaining CPU lifetime $r_{j}$. Furthermore, $\pi_{j}$ is inversely proportional to the total available memory space $M_{i}$. Therefore, the number of page faults $\pi_{j}$ of task $\tau_{j}$ can be written as $\pi_{j}=p_{j} \cdot \frac{L_{i}^{m e m}}{M_{i}} \cdot r_{j}$. The I/O processing time $\mu$ of each page fault is the summation of the seek time $t_{\text {seek }}$, the rotational latency $t_{\text {rot }}$, and the data transfer time 
Journal of Network and Computer Applications, vol. 31, no. 1, pp. 32-46, January 2008.

$\frac{d_{\text {page }}}{B_{\text {disk }}}$. Thus, the I/O processing time $\mu$ is expressed by $\mu=t_{\text {seek }}+t_{\text {rot }}+\frac{d_{\text {page }}}{B_{\text {disk }}}$. Hence, the page fault processing time $t_{j}^{\text {page }}$ is written as follows if $L_{i}^{m e m}>M_{i}$

$$
\begin{aligned}
t_{j}^{\text {page }} & =\pi_{j} \cdot \mu=p_{j} \cdot \frac{L_{i}^{\text {mem }}}{M_{i}} \cdot r_{j} \cdot \mu \\
& =p_{i} \cdot \frac{\sum_{j \in N_{i}} L_{j, m e m}}{M_{i}} \cdot r_{j} \cdot\left(t_{\text {seek }}+t_{\text {rot }}+\frac{d_{\text {page }}}{B_{\text {disk }}}\right),
\end{aligned}
$$

which completes the proof of the theorem 3.

Theorem 5. Let $\tau_{j}$ denote a task to be allocated in a cluster. For each node $N_{i}$ in a cluster, if the total available memory space $M_{i}$ is able to meet the memory demands of $\tau_{j}$ and tasks running on $N_{i}$ (i.e., $L_{j, m e m}+L_{i}^{m e m} \leq M_{i}$ ), then the allocation of task $\tau_{j}$ has no impact on balancing memory resources.

Proof. It is proved that $L_{i}^{m e m} \leq M_{i}$, since we have $L_{j, m e m}+L_{i}^{m e m} \leq M_{i}$ for each node $N_{i}$ in the cluster. In light of Theorem 3, we can prove that before the allocation of $\tau_{j}$, it is true that $\forall \tau_{k} \in N_{i}: t_{k}^{\text {page }}=0$. Hence, we have $\forall 1 \leq i \leq n: L_{i}^{\text {page }}=\sum_{\tau_{k} \in N_{i}} t_{k}^{\text {page }}=0$. The amount of imbalance with respect to memory resources can be measured by $\sum_{i=1}^{n}\left|L_{i}^{\text {page }}-\bar{L}_{i}^{\text {page }}\right|$, where $\bar{L}_{i}^{\text {page }}=\frac{1}{n} \sum_{i=1}^{n} L_{i}^{\text {page }}$. Prior to the allocation of $\tau_{j}$, we have $\sum_{i=1}^{n}\left|L_{i}^{\text {page }}-\bar{L}_{i}^{\text {page }}\right|=0$. Similarly, after the allocation of $\tau_{j}$, we have $\forall 1 \leq i \leq n: L_{i}^{\text {page }}=\sum_{\tau_{k} \in N_{i}} t_{k}^{\text {page }}=0$, because $L_{j, m e m}+L_{i}^{\text {mem }} \leq M_{i}$. Consequently, the value of $\sum_{i=1}^{n}\left|L_{i}^{\text {page }}-\bar{L}_{i}^{\text {page }}\right|$ is still zero after the allocation of $\tau_{j}$, meaning that 
Journal of Network and Computer Applications, vol. 31, no. 1, pp. 32-46, January 2008.

the allocation of $\tau_{j}$ has no impact on balancing memory resources.

Before proceed to the proof of a necessary condition for allocating tasks such that the global usage of memory resources in a cluster is improved, we first prove the following two lemmas.

Lemma 1. Suppose there is a task $\tau_{j}$ (initially submitted to node $N_{i}$ ) to be allocated in a cluster; the page fault processing time of $\tau_{j}$ on $N_{i}$ is $t_{j}^{\text {page }}$. If the page fault processing time $t_{j}^{\text {page }}$ of $\tau_{j}$ on $N_{i}$ equals to zero, then the page fault processing times of all tasks running on $N_{i}$ equal to zero. More formally, we have $t_{j}^{\text {page }}=0 \rightarrow \forall \tau_{k} \in N_{i}: t_{k}^{\text {page }}=0$.

Proof. Let $L_{i}^{m e m}$ be the accumulative memory space allocated to tasks running on $N_{i}$ prior to the allocation of $\tau_{j}$. Since $t_{j}^{\text {page }}$ equals to zero, we show that the sum of $L_{j, m e m}$ and $L_{i}^{m e m}$ is smaller than the total available memory space in $N_{i}$ (i.e., $L_{j, m e m}+L_{i}^{m e m} \leq M_{i}$ ). Hence, we have $L_{i}^{m e m} \leq M_{i}$, meaning that the page fault processing time of each task running on $N_{i}$ is zero (i.e., $\left.\forall \tau_{k} \in N_{i}: t_{k}^{\text {page }}=0\right)$. Hence, the proof.

Lemma 2. Suppose there is a task $\tau_{j}$ (initially submitted to node $N_{i}$ ) to be allocated in a cluster; the page fault processing time of $\tau_{j}$ on $N_{i}$ is $t_{j}^{\text {page }}$. If the page fault processing time $t_{j}^{\text {page }}$ of $\tau_{j}$ on $N_{i}$ equals to zero, then the memory load index $L_{i}^{\text {page }}$ of node $i$ equals to zero (i.e., $L_{i}^{\text {page }}=0$ ).

Proof. The memory load index $L_{i}^{\text {page }}$ is measured by $L_{i}^{\text {page }}=\sum_{\tau_{k} \in N_{i}} t_{k}^{\text {page }}$. As per Lemma 1 , it is proved that the page fault processing time of all tasks running on $N_{i}$ are zero (i.e., $\left.\forall \tau_{k} \in N_{i}: t_{k}^{\text {page }}=0\right)$. Therefore, we have $L_{i}^{\text {page }}=\sum_{\tau_{k} \in N_{i}} t_{k}^{\text {page }}=0$. 
Journal of Network and Computer Applications, vol. 31, no. 1, pp. 32-46, January 2008.

Theorem 6. Suppose there is a task $\tau_{j}$ (initially submitted to node $N_{i}$ ) to be allocated in a cluster; the page fault processing time of $\tau_{j}$ on $N_{i}$ is $t_{j}^{\text {page }}$. Then $t_{j}^{\text {page }}>0$ is a necessary condition for allocating task $\tau_{j}$ in a way to balance load in terms of memory resources.

Proof. The proof of Theorem 5 is immediate from Lemma 2. To prove the correctness of Theorem 5, we have to show that allocating $\tau_{j}$ in a way to balance load in memory resource implies that $t_{j}^{\text {page }}$ is larger than 0 . This can be proved by contradiction and, hence, let us assume that $t_{j}^{\text {page }}$ equals to 0 . Since $t_{j}^{\text {page }}=0$, Lemma 2 shows that the load index with respect to memory resource is 0 , implying that there is no way of allocating $\tau_{j}$ such that load in memory resource is balanced. Hence, we obtain the contradiction that completes the proof.

\subsection{A qualitative comparison}

Now we qualitatively compare the IOLB algorithm with two existing scheduling approaches: the CPU-based load-balancing algorithm (hereinafter referred to as CLB) [12] and the memorybased load-balancing algorithm (hereinafter referred to as MLB) [32]. The CLB load-balancing policy strives to improve global usages of CPU resources by balancing CPU load across all nodes in a cluster. The MLB load-balancing policy is conducive to balancing workload with respect to memory resources in a cluster when the cluster experiences a large number of page faults due to insufficient memory space. Both CLB and MLB are respectively concerned with effective usages of global CPU and memory resources in clusters without addressing the issue of balancing disk I/O resources. Consequently, the existing load-balancing approaches become inadequate for mixed workloads with CPU-, memory-, and I/O-intensive applications.

Theorem 7. Suppose there is a task $\tau_{j}$ (initially submitted to node $i$ ) to be allocated in a cluster; 
Journal of Network and Computer Applications, vol. 31, no. 1, pp. 32-46, January 2008.

node $k$ has the lightest load in disk I/O (i.e., $L_{k}^{I O}=\min _{a=1}^{n}\left(L_{a}^{I O}\right)$ ). If the following five conditions are satisfied, then the IOLB algorithm outperforms the CLB and MLB algorithms.

$$
\begin{aligned}
& \text { (1) } c_{j} \cdot \lambda_{j} \cdot t_{j, I O}>0, \text { (2) } L I F_{I O}=\max \left(L I F_{C P U}, L I F_{p a g e}, L I F_{I O}\right), \text { (3) } L_{i}^{I O}=\max _{a=1}^{n}\left(L_{a}^{I O}\right), \\
& c_{j} \cdot \lambda_{j} \cdot t_{j, I O}<\left(c_{j} \cdot \lambda_{j} \cdot t_{j, I O}+L_{i}^{I O}-L_{k}^{I O}\right) / 2 \text {, and (5) } R_{j}^{i}>R_{j}^{k}+e .
\end{aligned}
$$

Proof. First, theorem 3 shows that $c_{j} \cdot \lambda_{j} \cdot t_{j, I O}>0$ is a necessary condition for balancing disk I/O load. Second, $L I F_{I O}=\max \left(L I F_{C P U}, L I F_{p a g e}, L I F_{I O}\right)$ indicates that balancing disk I/O load can achieve more performance improvement than balancing memory or CPU resources. Third, $L_{i}^{I O}=\max _{a=1}^{n}\left(L_{a}^{I O}\right)$ means that the local node is overly loaded in terms of disk I/O. Since both the CLB and MLB algorithms do not take disk I/O load into account, leaving disk I/O resources severely imbalanced under conditions (1), (2), and (3). the suffer significant performance drop under I/O-intensive workload due to the imbalance of I/O load. Furthermore, condition (4) guarantees that allocating task $\tau_{j}$ to node $\mathrm{k}$ with the lightest disk I/O load can efficiently alleviate the imbalance problem, whereas condition (5) ensures that the expected response time of $\tau_{j}$ on the candidate remote node $k$ is less than the response time of $\tau_{j}$ on the local node $i$. Consequently, if the above conditions hold, then the IOLB algorithm outperforms the CLB and MLB algorithms.

Theorem 8. If each task $\tau_{j}$ running on a cluster imposes no load on disk resources (i.e., $\left.c_{j} \cdot \lambda_{j} \cdot t_{j, I O}=0\right)$, then the IOLB algorithm degrades to the MLB algorithm.

Proof. If the value of $c_{j} \cdot \lambda_{j} \cdot t_{j, I O}$ for each task $\tau_{j}$ is 0 , the first condition in Step 3 in the IOLB 
Journal of Network and Computer Applications, vol. 31, no. 1, pp. 32-46, January 2008.

algorithm does not hold, then Steps 4-8 used to balance disk I/O load across disks in the cluster are not performed by IOLB. In this case the IOLB algorithm strives to balance load with respect to memory resources if the memory load exceeds the amount of available memory space. This means that the behavior of IOLB is the same as that of MLB if the value of $c_{j} \cdot \lambda_{j} \cdot t_{j, I O}$ for each task $\tau_{j}$ is 0 . Hence, in case that each task $\tau_{j}$ running on a cluster imposes no load on disk resources (i.e., $c_{j} \cdot \lambda_{j} \cdot t_{j, I O}=0$ ), the performance of IOLB and MLB are identical. This completes the proof of Theorem 5 .

Theorem 9. If each task $\tau_{j}$ running on a cluster imposes no load on disk and memory resources (i.e., $c_{j} \cdot \lambda_{j} \cdot t_{j, I O}=0$ and $t_{j}^{\text {page }}=0$ ), then the IOLB algorithm degrades to the CLB algorithm.

Proof. First, if the value of $c_{j} \cdot \lambda_{j} \cdot t_{j, I O}$ for each task $\tau_{j}$ is 0 , the first condition in Step 3 in the IOLB algorithm does not hold, then Steps 4-8 used to balance disk I/O load across disks in the cluster are not performed by IOLB. Second, if the page fault processing time $t_{j}^{\text {page }}$ of $\tau_{j}$ is zero, Steps 9-14 are not invoked to improve the global usage of memory resources in the cluster. In this case the IOLB algorithm makes an effort to evenly distribute the CPU load. Therefore, the behavior of IOLB is the same as that of CLB. This completes the proof of Theorem 8.

\section{Experimental Results}

In this section we quantitatively compare the IOLB algorithm with the two existing loadbalancing approaches (see Section 4.2): CLB and MLB. We conducted trace-driven simulations using a simulated cluster with 32 nodes providing a time-sharing environment. A similar simulation environment was delineated in [23]. The traces used in our experiments were 
Journal of Network and Computer Applications, vol. 31, no. 1, pp. 32-46, January 2008.

extrapolated from those traces reported in [12][32]. The number of tasks of each parallel job in traces is selected randomly with a uniform distribution between 2 to 32. CPU times and memory demands of jobs are specified in the traces. We assume that the disk request arrival rate of each job is generated randomly with a uniform distribution. This assumption is reasonable because the mean disk request arrival rate can be manipulated and examined as a system parameter. In our empirical studies, we varied the mean disk request arrival rate from 0.8 to $1.25 \mathrm{No.} / \mathrm{ms}$. The data size of disk requests in each job is selected randomly based on a Gamma distribution with the mean size of $256 \mathrm{Kbyte}$. The performance metric by which we evaluate system performance is mean slowdown of all the jobs in a trace.

Table 1. Mean slowdowns of parallel jobs under the CLB, MLB, and IOLB schemes.

\begin{tabular}{crrrrrrrrrr}
\hline$\lambda$ & 0.80 & 0.85 & 0.90 & 0.95 & 1.0 & 1.05 & 1.10 & 1.15 & 1.20 & 1.25 \\
\hline CLB & 62 & 74 & 86 & 99 & 113 & 132 & 149 & 168 & 187 & 210 \\
MLB & 62 & 75 & 87 & 100 & 114 & 132 & 149 & 171 & 192 & 218 \\
IOLB & 47 & 61 & 73 & 80 & 94 & 101 & 117 & 130 & 151 & 172 \\
\hline
\end{tabular}

The number of nodes in the simulated cluster is 32 ; number of tasks in each job is selected randomly with a uniform distribution between 2 to 32; disk request arrival rate is varied from 0.8 to $1.25 \mathrm{No} . / \mathrm{ms}$; disk request size is chosen randomly with a Gamma distribution with mean size of $256 \mathrm{Kbyte}$; the page fault rate is set to $0.5 \mathrm{No} . / \mathrm{ms}$; the page size is $4 \mathrm{KBy}$ te.

Table 1 shows impacts of the disk request arrival rate on the mean slowdowns of submitted parallel jobs under the three evaluated load-balancing schemes. It is intuitive that regardless of the load-balancing approaches, the slowdowns of the parallel jobs increase with the I/O load goes up. This is because high disk request arrival rates leads to high disk I/O loads, which in turn cause long I/O processing time and long waiting time on disk I/O resources. More importantly, the experimental results reveal that the IOLB algorithm is superior to the CLB and MLB loadbalancing schemes. These results indicate that the existing load-balancing policies are inadequate for I/O-intensive workloads. The performance improvements can be attributed to the fact that 
Journal of Network and Computer Applications, vol. 31, no. 1, pp. 32-46, January 2008.

CLB and MLB do not address the issue of balancing disk I/O load under I/O-intensive workload conditions.

Table 2. Mean slowdowns of parallel jobs under the CLB, MLB, and IOLB schemes.

\begin{tabular}{lrrrrrrrrrr}
\hline page fault rate & 2.6 & 2.7 & 2.8 & 2.9 & 3 & 3.1 & 3.2 & 3.3 & 3.4 & 3.5 \\
\hline CLB & 22 & 23 & 23 & 24 & 24 & 24 & 24 & 27 & 27 & 28 \\
MLB & 8.5 & 8.6 & 8.8 & 10.8 & 10.9 & 11.1 & 13 & 13.5 & 14.2 & 14.3 \\
IOLB & 8.5 & 8.5 & 8.7 & 10.8 & 10.9 & 11 & 13.1 & 13.5 & 14.2 & 14 \\
\hline
\end{tabular}

Disk request arrival rate is fixed to $0.01 \mathrm{No} . / \mathrm{ms}$; the page fault rate varies from 2.6 to 3.5 No./ms; the page size is $4 \mathrm{KByte}$.

Recall that Theorem 8 proves that if all parallel jobs running on a cluster impose no load on disk I/O resources, then the performance of IOLB is identical to that of MLB. Now we experimentally validate the correctness of Theorem 8 using memory-intensive workloads. To achieve this goal, we varied the page fault rate from 2.6 to 3.5 No./ms with an increment of 0.1 No./ms. The disk request arrival rate is fixed to a low value -0.01 No./ms.

Table 2 shows performance impacts of the page fault rate on the mean slowdowns of parallel jobs running on the simulated cluster. For all the three examined load-balancing schemes, results in Table 2 indicate that MLB and IOLB outperform CLB under memory-intensive workloads. These performance improvements are possible because MLB and IOLB are concerned with the global memory usage in the cluster by balancing memory resources. The improved memory usage in turn significantly reduces time spent in page fault processing. This trend becomes more pronounced when the page fault rate is increased.

\section{Conclusions}

Most existing load balancing approaches are inadequate for $\mathrm{I} / \mathrm{O}$-intensive workloads due to imbalance of I/O loads and low usage of global disk resources. To address this issue, in this paper we proposed a new load-balancing algorithm (referred to as IOLB) for clusters. The 
Journal of Network and Computer Applications, vol. 31, no. 1, pp. 32-46, January 2008.

proposed load-balancing algorithm aims to achieve the effective usage of global disk resources in a cluster. This can, in turn, minimize the average slowdown of all parallel jobs running on a cluster and reduce the average response time of the jobs. In addition to balancing loads in disk resources under I/O-intensive workloads, the IOLB algorithm improves the CPU and memory utilization under CPU- and memory-intensive workload conditions. Consequently, IOLB is able to maintain the same level of performance as two existing CPU- and memory-aware loadbalancing schemes. We conducted trace-driven simulations where traces are composed of parallel applications with a wide variety of I/O demands. Empirical results demonstratively show that compared with the two existing load-balancing approaches, the IOLB algorithm significantly improves the resource utilization of a cluster under I/O-intensive workloads. When the workloads become CPU-intensive or memory-intensive in nature, IOLB gracefully degrades towards the existing load-balancing schemes.

Future studies can be performed in the following directions. First, we will evaluate the performance of IOLB on a large-scale cluster with more than 1000 nodes. Second, in this study we assume that network communication cost is negligible. Therefore, we intend to further extend our load-balancing algorithm in a way to balance load in network resources. Third, a heterogeneity-aware load-balancing algorithm will be investigated to deal with parallel jobs running on heterogeneous clusters, in which nodes have various processing capabilities.

\section{Acknowledgements}

The work reported in this paper was supported by the US National Science Foundation under Grants No. CCF-0742187 and No. CNS-0713895, Auburn University under a startup grant, and the Intel Corporation under Grant No. 2005-04-070. 
Journal of Network and Computer Applications, vol. 31, no. 1, pp. 32-46, January 2008.

\section{References}

[1] A. Acharya and S. Setia, "Availability and Utility of Idle Memory in Workstation Clusters," Proc. ACM SIGMETRICS Conf. Measuring and Modeling of Computer Systems, May 1999.

[2] A. Acharya et al., "Tuning the Performance of I/O-intensive Parallel Applications," Proc. 4th IOPADS, pp. 15-27, Philadelphia, PA, May 1996.

[3] J.M. Bahi, S. Contassot-Vivier, R. Couturier, "Dynamic load balancing and efficient load estimators for asynchronous iterative algorithms," IEEE Trans. Parallel and Distributed Systems, vol. 16, no. 4, pp. 289-299, April 2005.

[4] J. Basney and M. Livny, "Managing Network Resources in Condor", Proc. IEEE Symp. High Performance Distributed Computing, pp 298-299, Aug. 2000.

[5] B. Bode, D. M. Halstead, R. Kendall, and Z. Lei, "The Portable Batch Scheduler and the Maui Scheduler on Linux Clusters," Proc. 4th Annual Linux Showcase \& Conference, 2000.

[6] C. Chang, B. Moon, A. Acharya, C. Shock, A. Sussman, and J. Saltz, "Titan: a HighPerformance Remote-Sensing Database," Proc. 13th Int'l Conf. Data Eng., Apr. 1997.

[7] J. Choi, S.H. Noh, S.L. Min, and Y. Cho, "Design, Implementation, and Performance Evaluation of a Detection-Based Adaptive Block Replacement Scheme," IEEE Trans. Computers, vol. 51, no. 7, July 2002.

[8] J. Cruz and Kihong Park, "Towards Communication-Sensitive Load Balancing," Proc. 21 Int'l Conf. Distributed Computing Systems, Apr. 2001.

[9] A.C. Dusseau, R.H.Arpaci, and D.E. Culler, "Effective Distributed Scheduling of Parallel Workload," Proc. ACM SigMetrics Conf. Measuring and Modeling of Computer Systems, pp.25-36, May 1996.

[10] S.M. Figueira and F. Berman, "A Slowdown Model for Applications Executing on TimeShared Clusters of Workstations," IEEE Trans. Parallel and Distributed Systems, vol. 12, no. 6, pp. 653-670, June 2001.

[11] B. Forney, A. C. Arpaci-Dusseau, and R. H. Arpaci-Dusseau, "Storage-Aware Caching: Revisiting Caching for Heterogeneous Storage Systems," Proc. 1st File and Storage Technology, 2001. 
Journal of Network and Computer Applications, vol. 31, no. 1, pp. 32-46, January 2008.

[12] M. Harchol-Balter and A. Downey, "Exploiting Process Lifetime Distributions for Load Balacing," ACM Trans. Computer Systems, vol. 3, no. 31, 1997.

[13] B. Hendrickson and D. Womble, "The torus-wrap mapping for dense matrix calculations on massively parallel computers," SIAM J. Sci. Comput., vol. 15, no. 5, Sept. 1994.

[14] C. Hui and S. Chanson, "Improved Strategies for Dynamic Load Sharing," IEEE Concurrency, vol. 7, no. 3, 1999.

[15] R. Lavi and A. Barak, "The Home Model and Competitive Algorithm for Load Balancing in a Computing Cluster," Proc. Int'l Conf. Distributed Computing Systems, Apr. 2001.

[16] L. Lee, P. Scheauermann, and R. Vingralek, "File Assignment in Parallel I/O Systems with Minimal Variance of Service time," IEEE Trans. Computers, vol. 49, no.2, pp.127-140, 2000.

[17] J.-M. Liu X.-L. Jin, and Y.-S. Wang, "Agent-based load balancing on homogeneous minigrids: macroscopic modeling and characterization," IEEE Trans. Parallel and Distributed Systems, vol. 16, no. 7, pp. 586-598, July 2005.

[18] X. Ma, M. Winslett, J. Lee, and S. Yu, "Faster Collective Output through Active Buffering," Proc. Int'l Symp. Parallel and Distributed Processing, 2002.

[19] A. Mueller, "Fast sequential and parallel algorithms for association rule mining: A comparison," Technical Report CS-TR-3515, University of Maryland, College Park, Aug. 1995.

[20] B.K. Pasquale and G.C. Polyzos. "Dynamic I/O Characterization of I/O Intensive Scientific applications," Proc. Int'l Conf. Supercomputing, pp. 660-669, 1994.

[21] X. Qin, H. Jiang, Y. Zhu, and D. Swanson, "Dynamic Load balancing for I/O- and Memory-Intensive workload in Clusters using a Feedback Control Mechanism," Proc. 9th Int'l Euro-Par Conf. Parallel Processing, Klagenfurt, Austria, Aug. 2003.

[22] X. Qin, H. Jiang, Y. Zhu, and D.R. Swanson, "Improving the Performance of I/O-Intensive Applications on Clusters of Workstations," Cluster Computing: The Journal of Networks, Software Tools and Applications, vol. 8, no. 4, Oct. 2005.

[23] X. Qin, H. Jiang, Y. Zhu, and D. R. Swanson, “Towards Load Balancing Support for I/OIntensive Parallel Jobs in a Cluster of Workstations," Proc. 5th IEEE Int'l Conf. Cluster Computing, pp.100-107, Dec. 2003. 
Journal of Network and Computer Applications, vol. 31, no. 1, pp. 32-46, January 2008.

[24] X. Qin and H. Jiang, "Improving Effective Bandwidth of Networks on Clusters using Load Balancing for Communication-Intensive Applications," Proc. 24th IEEE Int'l Performance, Computing, and Communications Conf., pp.27-34, April 2005.

[25] J.O. Roads, et al., "A Preliminary Description of the Western U.S. Climatology", Proc. 9th Annual Pacific Climate Workshop, Sept. 1992.

[26] M. Surdeanu, D. Modovan, and S. Harabagiu, "Performance Analysis of a Distributed Question/ Answering System," IEEE Trans. Parallel and Distributed Systems, vol. 13, No. 6, pp. 579-596, 2002.

[27] M. Uysal, A. Acharya, and J. Saltz. "Requirements of I/O Systems for Parallel Machines: An Application-driven Study," Technical Report, CS-TR-3802, University of Maryland, College Park, May 1997.

[28] G. Voelker, "Managing Server Load in Global Memory Systems," Proc. ACM SIGMETRICS Conf. Measuring and Modeling of Computer Systems, May 1997.

[29] B. Wilkinson, and M. Allen, "Parallel Programming, Techniques and Applications Using Networked Workstations and Parallel Computers", Prentice-Hall, Inc., 1999.

[30] S. Wu and U. Manber, "agrep - A fast approximate pattern-matching tool," USENIX Conference Proc., pp. 153-162, San Francisco, CA, Winter 1992.

[31] C.-Z Xu, B. Monien, and R. Luling, "An Analytical Comparison of Nearest Neighbor Algorithms for Load Balancing in Parallel Computers," Proc. Int'l Symp. Parallel Processing, 1995.

[32] X. Zhang, Y. Qu, and L. Xiao, "Improving Distributed Workload Performance by Sharing both CPU and Memory Resources," Proc. 20th Int'l Conf. Distributed Computing Systems, Apr. 2000.

[33] Y. Zhang, A. Yang, A. Sivasubramaniam, and J. Moreira, "Gang Scheduling Extensions for I/O Intensive Workloads," Proc. 9th Workshop Job Scheduling Strategies for Parallel Processing, 2003. 